\title{
Parazentese - Peritonealpunktion - Aszitespunktion: Schritt für Schritt
}

\author{
Ulrich Brauckmann, Kyung-A Na, Daniel Jaspersen
}

\section{Grundlagen}

\section{Ziel und Zweck}

Im Rahmen der Aszitespunktion oder Peritonealpunktion wird die Bauchhöhle zur Gewinnung dort befindlicher freier Flüssigkeit für diagnostische und/oder therapeutische Zwecke punktiert. Das Auftreten von freier Flüssigkeit in der Peritonealhöhle signalisiert in der Regel eine schwere Erkrankung. Je nach Ausprägung von Krankheitsbild und Symptomatik ist die rasche Aszitespunktion zur differenzialdiagnostischen Abklärung und Einleitung therapeutischer Konsequenzen indiziert.

Meist handelt es sich bei freier Flüssigkeit im Bauchraum um Aszites hepatischer, kardialer, renaler oder maligner Genese, seltener um ein Hämatoperitoneum (z. B. nach abdominellem Trauma oder Operationen bzw. Tumorblutungen in die freie Bauchhöhle). Leitsymptomatik ist dabei häufig das manifeste akute Abdomen durch die peritoneale Reizung sowie gegebenenfalls eine hämodynamische Instabilität.

Die Peritonealpunktion als invasive Untersuchungstechnik hat in der Notaufnahme und auf der Intensivstation eine (deutlich) größere Bedeutung als in der prähospitalen Anwendung. Innerklinisch hat das Verfahren aufgrund des diagnostischen und therapeutischen Stellenwerts eine große Relevanz, beispielsweise zur Symptomlinderung (z. B. bei ausgeprägtem Spannungsaszites mit abdominellen Schmerzen und Dyspnoe) oder zur Asservierung von Aszites für die mikrobiologische Diagnostik (z. B. bei Sepsis bzw. bei V.a. Vorliegen einer spontanbakteriellen Peritonitis [SBP]).

Die Anamnese des Patienten ist zur differenzialdiagnostischen Abgrenzung des Aszites häufig wegweisend $(\triangleright$ Tab. 1).

\section{Indikationen und Kontraindikationen}

\section{Indikationen}

Eine Peritonealpunktion ist in folgenden Fällen indiziert [1]:

- bei allen Patienten mit Leberzirrhose und neu aufgetretenen Komplikationen (z. B. bei Dekompensation, Hepatoenzephalopathie, Infektzeichen, Verschlechterung der Leberfunktionsparameter oder Retentionswerte, abdominellen Schmerzen), zum Aus-
Tab. 1 Ursachen freier Flüssigkeit in der Peritonealhöhle (Quelle: [1]).

\begin{tabular}{|c|c|}
\hline $\begin{array}{l}\text { Genese der Flüssig- } \\
\text { keitsansammlung }\end{array}$ & mögliche Ursachen \\
\hline hepatisch & $\begin{array}{l}\text { - Leberzirrhose } \\
\text { " akute Leberschädigung } \\
\text { " Budd-Chiari-Syndrom/sinusoidales } \\
\text { Obstruktionssyndrom }\end{array}$ \\
\hline maligne & $\begin{array}{l}\text { - Peritonealkarzinose } \\
\text { - Tumorarrosionsblutung }\end{array}$ \\
\hline kardial & $\begin{array}{l}\text { - Rechtsherzinsuffizienz } \\
\text { " globale Herzinsuffizienz } \\
\text { " Pericarditis constrictiva }\end{array}$ \\
\hline entzündlich & $\begin{array}{l}\text { " spontan-bakterielle Peritonitis (SBP) } \\
\text { " sekundäre Peritonitis (z. B. Darmperforation, } \\
\text { Peritonealtuberkulose) } \\
\text {. Pankreatitis }\end{array}$ \\
\hline biliär & - Gallenblasenperforation \\
\hline traumatisch & $\begin{array}{l}\text { - Hämatoperitoneum im Rahmen einer Leber-/ } \\
\text { Milzruptur, Aortenruptur, Gefäßarrosion durch } \\
\text { Pankreatitis } \\
\text { - Uroperitoneum }\end{array}$ \\
\hline postoperativ & $\begin{array}{l}\text { - Gallenfistel nach Gallenblasen-/-gangoperation } \\
\text { - Leberpunktion } \\
\text { - Lymphfistel } \\
\text { - Nieren- oder Harnleiteroperation }\end{array}$ \\
\hline osmotisch & $\begin{array}{l}\text { " Eiweißmangelzustände z. B. bei Nephrotischem } \\
\text { Syndrom, Hypoalbuminämie, andere Ursachen }\end{array}$ \\
\hline
\end{tabular}

schluss einer SBP, die undiagnostiziert mit einer erhöhten Letalität, je nach begleitenden Risikofaktoren, von $10-50 \%$ einhergeht,

- zur Differenzierung einer SBP von einer sekundären bakteriellen Peritonitis (z. B. Perforation im Bereich des Magen-Darm-Traktes oder bei abdominellen Abszessen), die nach Diagnostik in der Regel operiert werden muss, insbesondere, da die Letalität der unbehandelten sekundären bakteriellen Peritonitis bei rund $50-80 \%$ liegt,

- im Rahmen der diagnostischen Peritonealpunktion bei Patienten mit neu aufgetretener freier Flüssigkeit zur Klärung der Genese,

- zur therapeutischen Aszitespunktion bei respiratorischer Beeinträchtigung durch große Aszitesvolumina oder beim Spannungsaszites mit abdominellen Schmerzen, Übelkeit und Erbrechen, Verschlechterung inguinaler/umbilikaler Hernien. 
Eine Aszitespunktion muss als Notfalltechnik in einer Notaufnahme bzw. auf einer Intensivstation zu jeder Zeit von dem dort eingesetzten ärztlichen Personal sicher, adäquat und unmittelbar beherrscht und durchgeführt werden können.

\section{Kontraindikationen}

Für die Aszitespunktion bestehen folgende Kontraindikationen:

- absolut:

- keine

- relativ:

- disseminierte intravasale Koagulopathie. Dabei Nutzen-Risiko-Abwägung durch individuelle Risikostratifizierung und ggf. Substitution von Gerinnungsfaktoren (aber keine prophylaktische Substitution mit Fresh-Frozen-Plasma [FFP]!),

- eingeschränkter perkutaner Zugangsweg zum Aszites (z.B. Zystennieren, Hepatosplenomegalie, ausgeprägte Darmdistension, adhärente Darmschlingen an der Bauchwand [ $\vee$ Abb. 1], mangelnde Kooperation des Patienten),

- Niereninsuffizienz mit erhöhten Kreatininwerten (ab > $6 \mathrm{mg} / \mathrm{dl}$ ) und konsekutiver Blutungsgefahr (z.B. durch eine verminderte Thrombozytenfunktion).

\section{Komplikationen}

Schwerwiegende Komplikationen treten seit Einführung der sonografiegestützten diagnostischen Parazentese extrem selten auf. Bis zum routinemäßigen Einsatz des UItraschalls (US) lag die Komplikationsrate bei ca. 2,9\% [2].

Therapeutische Aszitespunktionen haben eine höhere Komplikationsrate, wobei auch hier die schwerwiegenden Komplikationen selten sind $(<1 \%$ lebensbedrohliche und $<5 \%$ leichtere, nicht lebensbedrohliche Komplikationen) $[3-6]$.

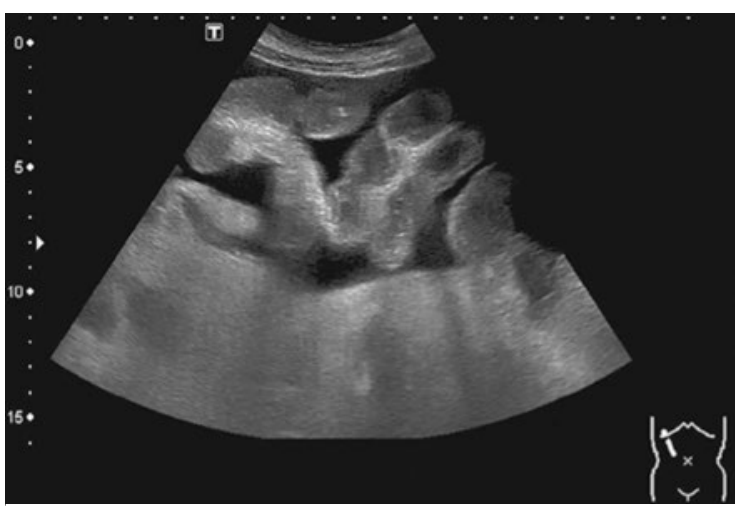

- Abb. 1 An der Bauchwand anliegende Darmschlingen. Durch Umlagerung kann zwischen adhärenten und frei schwimmenden Darmschlingen unterschieden werden.
Komplikationen der therapeutischen Parazentese:

- protrahierter Austritt von Aszites aus dem Stichkanal (bei 5\% der therapeutischen Parazentesen) [4],

- Peritonitis/Bauchdeckenabszess nach Darmperforation bei ca. 0,4\% der Parazentesen [2],

- Einblutungen in die Bauchdecke oder intraperitoneale Blutungen 0,2-1\% während der Parazentesen (Verletzungen epigastrischer Gefäße oder abdomineller Kollateralvenen) [5],

- kasuistisch postprozedurale Blutungen nach großvolumiger therapeutischer Parazentese (ohne Korrelation zu Koagulopathie/Thrombozytopenie) bis zu 7 Tage nach Parazentese [3],

- residuale abgerissene Katheterteile intraperitoneal/ intrakutan,

- lebensbedrohliche Blutungen nur vereinzelt beschrieben [6]. Selbst bei verlängerter PTT bis zum 2-Fachen des oberen Normwerts und einer Thrombozytopenie unter $50000 / \mu l$ stellt die Peritonealpunktion ein sicheres Verfahren dar. Eine Gerinnungsoptimierung durch Gabe von FFP/Thrombozytenkonzentraten ist bei einer INR > 2,5 oder Thrombozyten < 20000/ $\mu$ l laut Leitlinie zu erwägen. In der Klinik der Autoren erfolgt bei einer INR > 2,5 oder Thrombozyten < 20000/ $\mu \mathrm{l}$ die Gabe von FFP bzw. Thrombozytenkonzentraten.

- Ein Kreatininwert > 6 mg/dl wurde als Risikofaktor für Blutungskomplikationen beschrieben [7,8].

Postpunktionell kann es im Rahmen großvolumiger Parazentesen (> 5I) zum Blutdruckabfall kommen. Dann ist eine inter- und postinterventionelle Überwachung der Patienten inklusive der Vitalparameter angezeigt und sollte schon im Vorfeld geplant werden.

Die Gefahr des Auftretens eines hepatorenalen Syndroms bei großvolumigen Parazentesen ( $a b>5$ I) kann durch die intravenöse Humanalbuminsubstitution (6-8 g Albumin/l Aszites) vermieden werden [9].

Aus Praktikabilitätsgründen erhalten die Patienten in der Klinik der Autoren pro Liter abgelassenen Aszites $50 \mathrm{ml}$ einer 20\%igen Albuminlösung ( $10 \mathrm{~g}$ Albumin). Bei Aszitesmengen < 5 l ist keine Gabe von Albumin notwendig [9].

\section{Schritt für Schritt}

\author{
Merke \\ Nach den Richtlinien für Krankenhaushygiene und \\ Infektionsprävention soll die Aszitespunktion unter \\ sterilen Bedingungen erfolgen. Der Patient muss \\ über die Risiken der Maßnahme aufgeklärt werden. \\ Die Aszitespunktion sollte immer sonografisch ge- \\ stützt erfolgen, um Organverletzungen und frustrane \\ Punktionen ohne Aszitesgewinnung zu vermeiden.
}


Die Vorbereitung der Aszitespunktion umfasst folgende Arbeitsschritte:

- Vorbereiten und Funktionskontrolle des benötigten Materials

- Ermitteln des Punktionsorts

- Markieren der Punktionsstelle

- Abdecken des Punktionsareals und Desinfektion

Die weiteren Arbeitsschritte sind jeweils bei der diagnostischen und der therapeutischen Aszitespunktion angegeben.

\section{Schritt 1 Material}

Zur diagnostischen Aszitespunktion können dünnkalibrige Kanülen verwendet werden.

Bei therapeutischen Punktionen können Venenverweilkanülen, Pigtail-Katheter oder speziell für Aszitespunktionen entwickelte Punktionsnadeln mit mehreren Seitlöchern verwendet werden. Letztere haben den Vorteil einer hohen Knickstabilität und guter Befestigungsmöglichkeit an der Bauchdecke. Mehrere Seitlöcher gewährleisten meist ein kontinuierliches und vollständiges Drainieren des Aszites. Aus diesem Grund werden in der Klinik der Autoren überwiegend spezielle industriell gefertigte Punktionsnadeln verwendet (Nachteil: Kostenfaktor, Verfügbarkeit). Bei der Anwendung von Venenverweilkanülen müssen die Nachteile der höheren Knickinstabilität und der häufiger notwendigen Repositionierung (nur eine distale Öffnung) bedacht werden (Vorteile: Kostenfaktor, hohe Verfügbarkeit). Eine effektive oder suffiziente Aszitesentlastung gelingt häufig nicht.

Folgende Materialien werden zur Aszitespunktion benötigt:

- sterile Handschuhe

- steriles Lochtuch

- Lokalanästhesie (1\% Lidocain)

- Auffangbehälter für Punktionsmaterial

- 10-50-ml-Spritzen

- Punktionskanüle

- Bakterienkulturflaschen

- Röhrchen Blutbild und klinische Chemie

- Drainagebeutel

- Fixierungsmaterial

\section{Schritt 2 Ermitteln des Punktionsorts}

Die Punktionsstelle wird sonografisch ermittelt. Geeignete Punktionsstellen finden sich im linken oder rechten unteren lateralen Quadranten in leichter Schräglage zur Punktionsseite hin gedreht sowie auf einer gedachten Linie zwischen Bauchnabel und Spina iliaca superior anterior im lateralen Drittel ( $\triangleright$ Abb. 2).

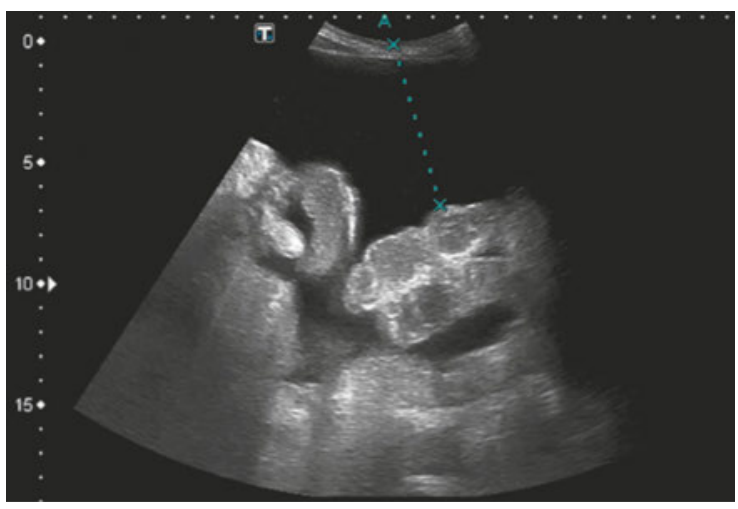

- Abb. 2 Echofreie Flüssigkeitsansammlung (z. B. hepatogener Aszites) im Peritoneum. Die Dünndarmschlingen sind frei umspült und bewegen sich wie Schlingpflanzen (sog. Seeanemonen-Phänomen).

Bei nur geringer Punktionsmenge kann auch unter direkter sonografischer Kontrolle punktiert werden oder in Knie-Ellenbogen-Position kaudal des Bauchnabels. Bei einer Punktionsstelle im rechten unteren Quadranten kann gelegentlich ein überblähtes Zökum oder eine Narbe nach Appendektomie eine sonografisch gestützte Punktion erschweren. Lässt sich sonografisch keine geeignete Stelle finden, so sollte im linken lateralen Unterbauch eine geeignete Stelle gesucht werden.

Berücksichtigt werden sollte bei der Wahl des Punktionsorts außerdem der mutmaßliche Verlauf der epigastrischen Gefäße und bei Vorhandensein von Kollateralvenen deren Verlauf.

\section{Schritt 3 Markieren der Punktionsstelle}

Die zuvor sonografisch infrage kommende Punktionsstelle wird markiert (hier im rechten Unterbauch liegend) ( Abb. 3).

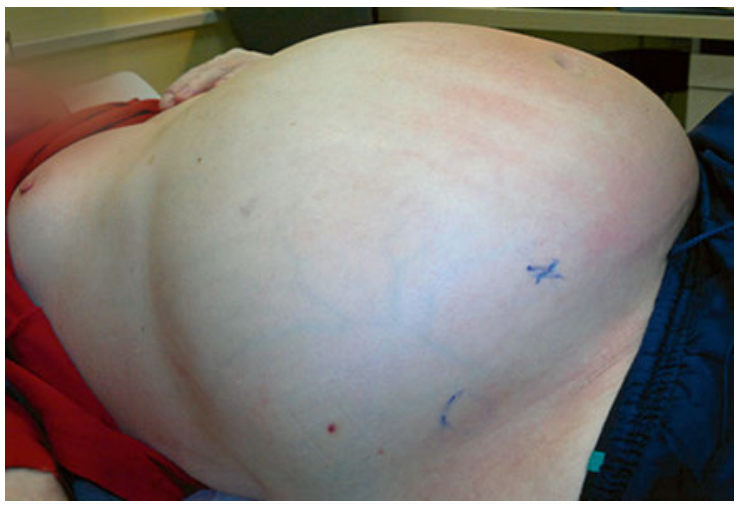

Abb. 3 Markierte Punktionsstelle. 


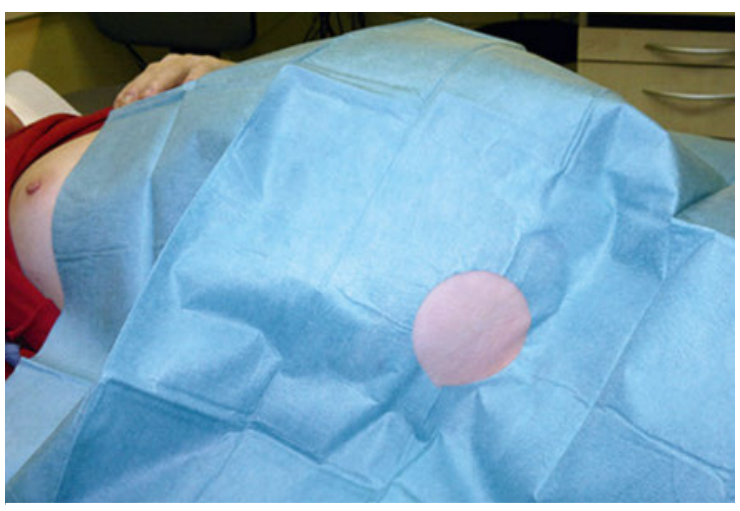

- Abb. 4 Abgedecktes Punktionsareal.
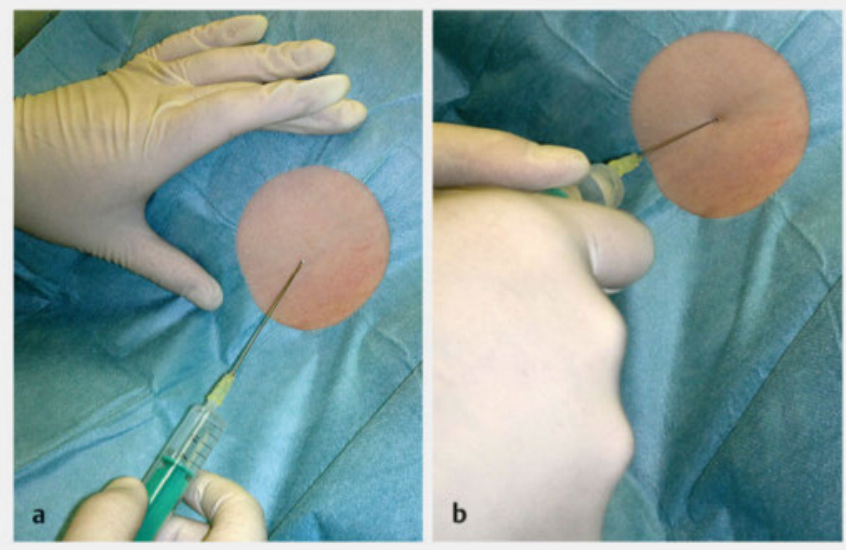

- Abb. 5 Straffung der Haut (a) und Aufsetzen der Punktionsnadel (b).
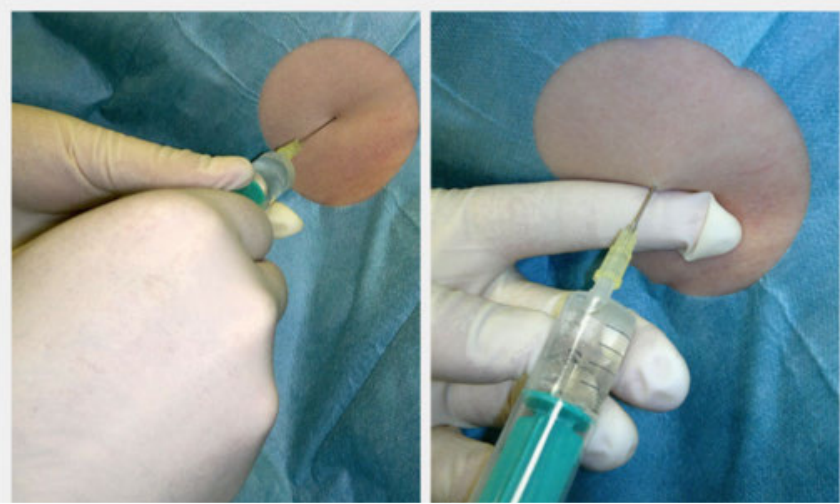

- Abb. 6 Vorschieben der Punktionsnadel (a) und Erreichen der Peritonealhöhle (b).

\section{Schritt 4 Abdecken des Punktions- areals und Desinfektion}

Anschließend erfolgt das sterile Abdecken des Punktionsareals ( $\triangleright$ Abb. 4 ) sowie die lokale Desinfektion mittels Hautantiseptikum (Einwirkzeit abwarten).

\section{Punktionstechnik}

Der Einstich erfolgt unter sterilen Bedingungen ohne vorherige Lokalanästhesie bei der diagnostischen und bevorzugt mit Lokalanästhesie bei der therapeutischen Punktion.

Die Punktion erfolgt unter Aspiration in einem Einstichwinkel von $45^{\circ}$ oder in Z-Durchstichtechnik zur Vermeidung einer Einstichfistel durch Retraktion der Haut nach kaudal vor und während der Punktion.

\section{Diagnostische Punktion}

Die diagnostische Aszitespunktion umfasst folgende Arbeitsschritte:

- Vorbereiten und Funktionskontrolle des benötigten Materials (s. „Material“)

- Ermitteln des Punktionsorts (s. „Ermitteln des Punktionsorts“)

- Markieren der Punktionsstelle (s. „Markieren der Punktionsstelle“)

- Abdecken des Punktionsareals und Desinfektion (s. „Abdecken des Punktionsareals und Desinfektion“)

- Vorbereiten der Punktion

- Aufsetzen der Punktionsnadel

- Vorschieben der Punktionsnadel

- Inspizieren des Aszites

\section{Schritt 5 Vorbereitung der Punktion und Aufsetzen der Punktionsnadel}

Für die Punktion wird eine zuvor mit 2-3 ml Kochsalzlösung gefüllte Spritze verwendet. Vor dem Aufsetzen der Nadel wird die Haut gestrafft ( $\bullet \mathbf{A b b} \mathbf{5} \mathbf{5}$ ).

Zur Verhinderung eines protrahierten Austritts von Aszites nach Punktion durch den Stichkanal wird ein schräger Stichkanal gewählt ( $\mathbf{A} \mathbf{b b} \mathbf{5} \mathbf{5}$ ) .

\section{Schritt 6 Vorschieben der Punktionsnadel und Erreichen der Peritonealhöhle}

Die Punktionsnadel wird unter leichtem Sog vorgeschoben ( $\bullet$ Abb.6a). Erster Aszites zeigt das Erreichen der Peritonealhöhle an ( $\triangleright$ Abb. $\mathbf{6 b}$ ). 


\section{Schritt 7 Inspektion des Aszites}

Die Inspektion des ersten Aszites ist wichtig zur Beurteilung, ob bereits initial hämorrhagischer Aszites vorliegt oder sich im weiteren Verlauf möglicherweise punktionsbedingt entwickelt hat ( $\triangleright$ Abb. 7).

Weitere Aspiration für diagnostische Zwecke und hierfür ggf. Befüllung weiterer Spritzen. Anschließend kann die Punktionsnadel gezogen werden.

Zum sicheren Verschluss sollte eine manuelle Kompression mit einem sterilen Tupfer für ca. 10 min erfolgen. Abschließend wird die Punktionsstelle mit einem sterilen Pflaster versorgt.

\section{DIAGNOSTIK AUS ASZITES BEI}

- V.a. spontan-bakterielle Peritonitis

- Bestimmung Zellzahl und Zelldifferenzierung

- segmentkernige Granulozyten

- Beimpfung von Blutkulturflaschen

- Gesamteiweiß im Aszites

- V.a. sekundäre bakterielle Peritonitis

- Eiweißgehalt im Aszites $>1 \mathrm{~g} / \mathrm{l}$

- Glukose <2,7 mmol/l

- Aszites-LDH oberhalb des Normbereichs der Serum-LDH

- V.a. hämorrhagischen Aszites

- Bestimmung des Hb-Wertes im Aszites

- V.a. malignen Aszites

- Zytologie

\section{Therapeutische Punktion}

Die therapeutische Aszitespunktion umfasst folgende Arbeitsschritte:

- Vorbereiten und Funktionskontrolle des benötigten Materials (s. „Material“)

- Ermitteln des Punktionsorts (s. „Ermitteln des Punktionsorts“)

- Markieren der Punktionsstelle (s. „Markieren der Punktionsstelle“)

- Abdecken des Punktionsareals und Desinfektion (s. „Abdecken des Punktionsareals und Desinfektion“)

- Lokalanästhesie

- Aufsetzen der Punktionsnadel

- Vorschieben der Punktionsnadel

- Rückziehen des Mandrins

- Vorschieben des Verweilkatheters

- Austreten von Aszites

- Anschließen des Drainagebeutels

- Lagekorrektur

- Abdecken der Punktionsstelle

- Fixieren der Drainage

- Ablaufen des Aszites

- Verarbeiten des Punktats und Diagnostik

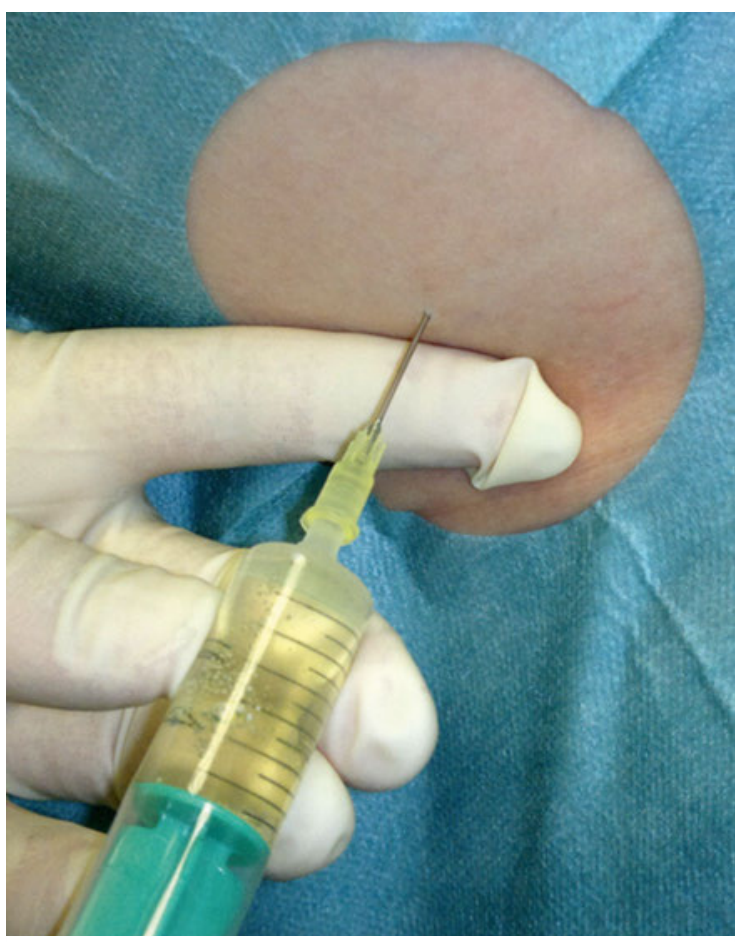

- Abb. 7 Inspektion des Aszites.

\section{Schritt 5 Lokalanästhesie und Aufsetzen der Punktionsnadel}

Das Lokalanästhetikum wird injiziert (z. B. Lidocain 1\%) ( $\bullet$ Abb. 8a). Zur Verhinderung eines protrahierten Austritts von Aszites nach der Punktion durch den Stichkanal wird ein schräger Stichkanal mit der Aszitespunktionsnadel gewählt ( $>$ Abb. 8 b).
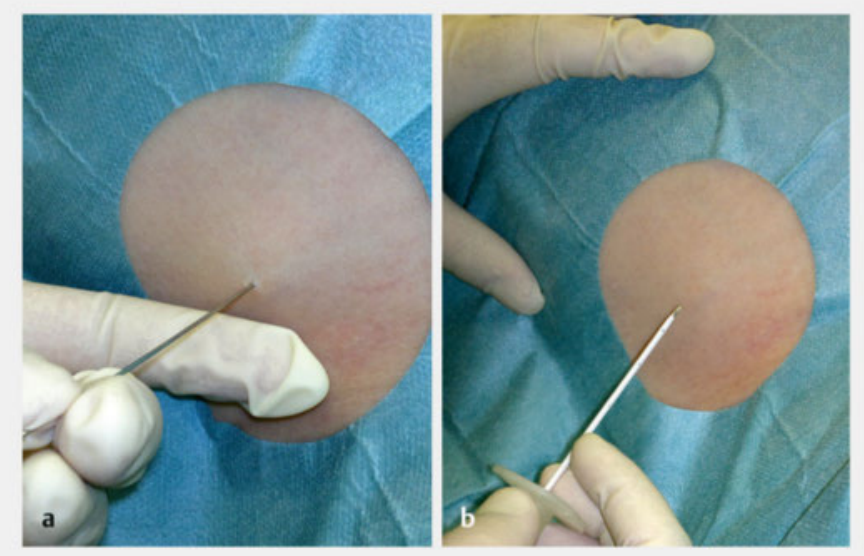

- Abb. 8 Injektion eines Lokalanästhetikums (z. B. Lidocain 1\%) (a) und Aufsetzen der Punktionsnadel (b). 


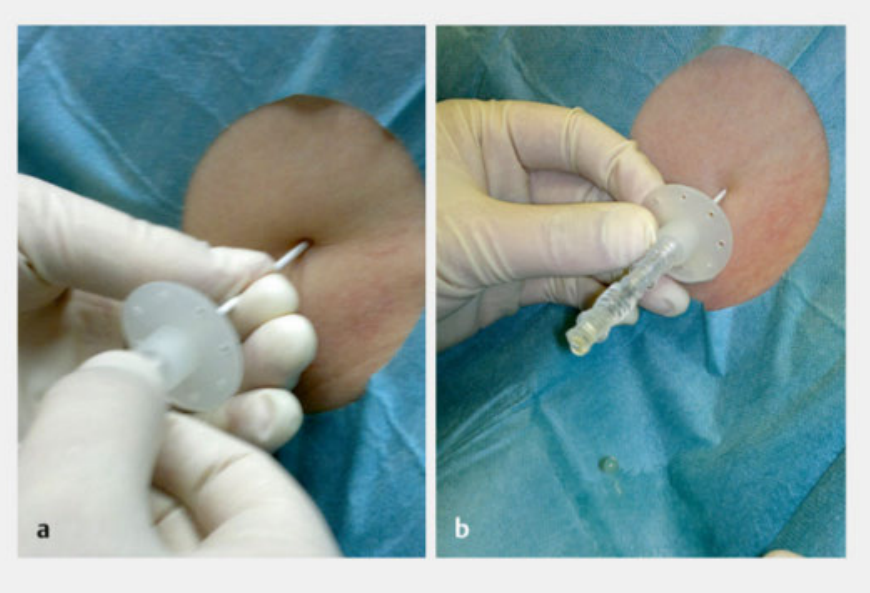

- Abb. 9 Vorschieben der Punktionsnadel (a) und Austritt von Aszites (b).

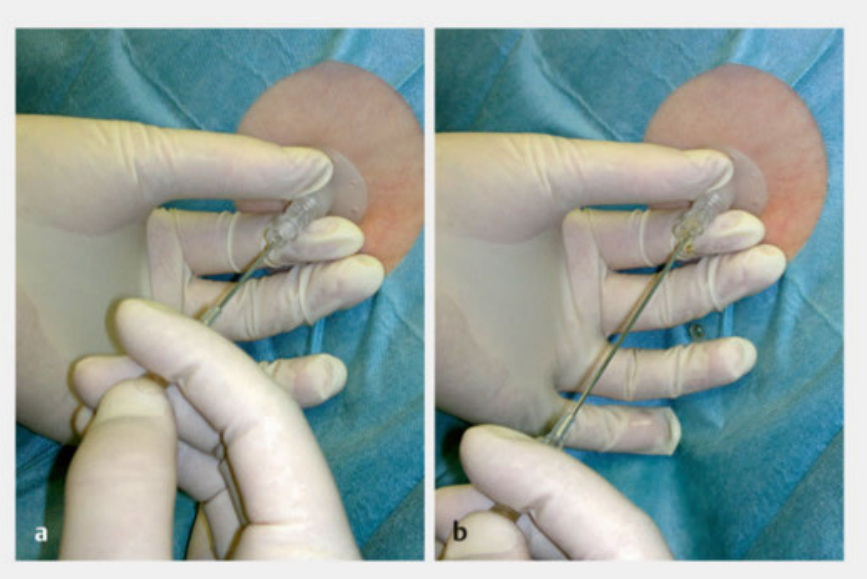

- Abb. 10 Rückzug des Mandrins (a) und Vorschieben des Verweilkatheters (b).
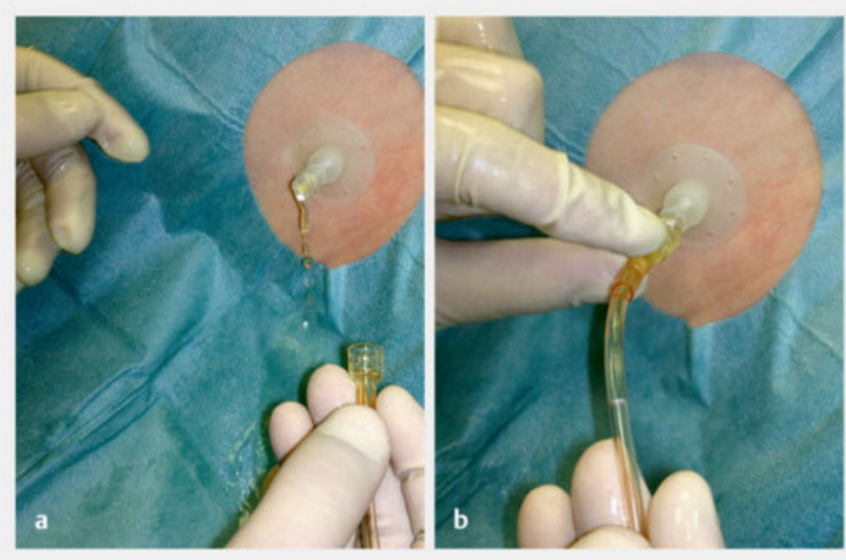

- Abb. 11 Spontaner Austritt von Aszites (a) und Anschluss des Drainagebeutels (b).

\section{Schritt 6 Vorschieben der Punk- tionsnadel und Austritt von Aszites}

Die Aszitespunktionsnadel wird durch die Haut- und Fettgewebsschichten vorgeschoben ( $\bullet$ Abb. 9a). Der Austritt von Aszites am Ende des Mandrins zeigt das Erreichen der Peritonealhöhle an ( $\mathbf{A} \mathbf{b b} \mathbf{9} \mathbf{9 b}$ ). Hierbei erfolgt die erste Beurteilung des Aszites (z. B. bereits initial hämorrhagischer Aszites oder erst im Verlauf punktionsbedingt entwickelt).

\section{Schritt 7 Zurückziehen des Mandrins und Vorschieben des Verweilkatheters}

Der Mandrin wird zurückgezogen ( $\vee$ Abb. 10 a). AnschlieBend wird der Verweilkatheter mit Halteplatte vorgeschoben und der Mandrin vollständig entfernt ( Abb. 10b).

\section{Schritt 8 Austreten von Aszites und Anschluss des Drainagebeutels}

Aufgrund des intraabdominellen Druckes tritt spontan Aszites aus ( $\triangleright$ Abb. 11a). Im nächsten Schritt wird das Drainagebeutelset angeschlossen ( $\bullet$ Abb. $11 \mathbf{b})$.

\section{Schritt 9 Lagekorrektur und Abdecken der Punktionsstelle}

Falls nötig, wird dann die Lage korrigiert ( $\bullet$ Abb. 12a). Die Punktionsstelle des intraabdominell verbleibenden Verweilkatheters wird auf Höhe der Halteplatte mit Schlitzkompressen steril abgedeckt ( $\triangleright$ Abb. 12 b).

\section{Schritt 10 Fixieren der Drainage und Ablaufen des Aszites}

Die Drainage wird mit Klebefolie fixiert ( $\bullet$ Abb. $13 a)$.

Der Aszites läuft in den Drainagebeutel ab ( $\bullet$ Abb. 13 b). Er wird erneut begutachtet und ggf. zu diagnostischen Zwecken weiterverwendet. Hierfür befindet sich bei einigen Sets ein Ablassventil am Beutelboden. Anschließend kann der Verweilkatheter gezogen werden. Ein Verbleib des Verweilkatheters über mehrere Tage sollte aufgrund des hohen Infektionsrisikos nicht erfolgen.

Zum sicheren Verschluss sollte eine manuelle Kompression mit einem sterilen Tupfer für ca. 10 min. erfolgen. Abschließend wird die Punktionsstelle mit einem sterilen Pflaster versorgt. 


\section{Schritt 11 Verarbeiten des Punktats und Diagnostik}

Die Gewinnung des Aszites ermöglicht eine gezielte ätiologische Diagnostik unter Berücksichtigung der Vorerkrankungen, Klinik, Anamnese und Dringlichkeit. Bei akutem Krankheitsbild steht die Differenzierung einer SBP zur sekundär bakteriellen Peritonitis und bei hämorrhagischem Aszites der Ausschluss einer akuten intraabdominellen Blutung im Vordergrund.

\section{Video}

Das Video 1 zeigt das Vorgehen bei der therapeutischen Aszitespunktion Schritt für Schritt.

\section{Erstveröffentlichung}

Dieser Beitrag wurde erstveröffentlicht in: Bernhard M, Gräsner J-T. Notfalltechniken Schritt für Schritt. Stuttgart: Thieme; 2016: 219-233

Autorinnen / Autoren

\section{Ulrich Brauckmann}

Dr. med., Klinikum Fulda, Medizinische Klinik II, Gastroenterologie

\section{Kyung-A Na}

Klinikum Fulda, Medizinische Klinik II, Gastroenterologie

\section{Daniel Jaspersen}

Prof. Dr. med., Klinikum Fulda, Medizinische Klinik II

\section{Korrespondenzadresse}

\section{Dr. med. Ulrich Brauckmann}

Klinikum Fulda

Medizinische Klinik II, Gastroenterologie

Pacelliallee 4

36043 Fulda

ulrich.brauckmann@klinikum-fulda.de
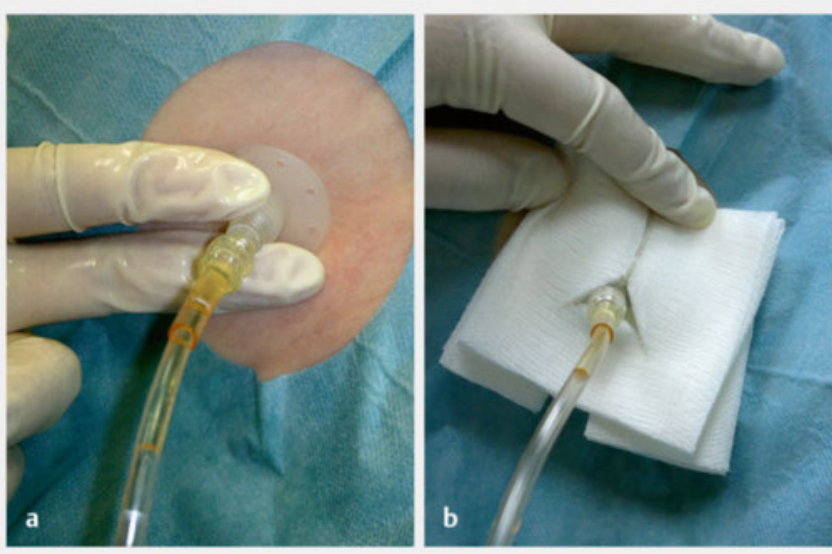

- Abb. 12 Ggf. Lagekorrektur (hier nicht erforderlich) (a) und steriles Abdecken der Punktionsstelle (b).

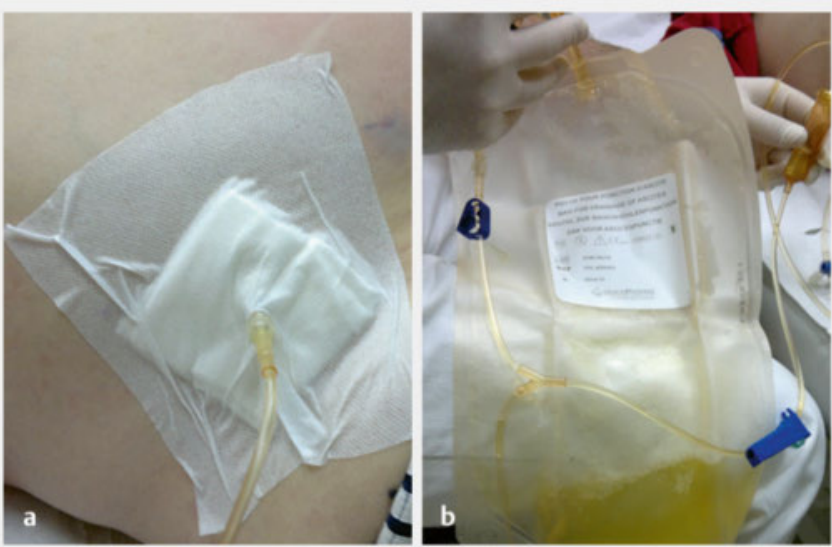

Abb. 13 Fixierte Drainage (a) und abgelaufener Aszites im Drainagebeutel (b).

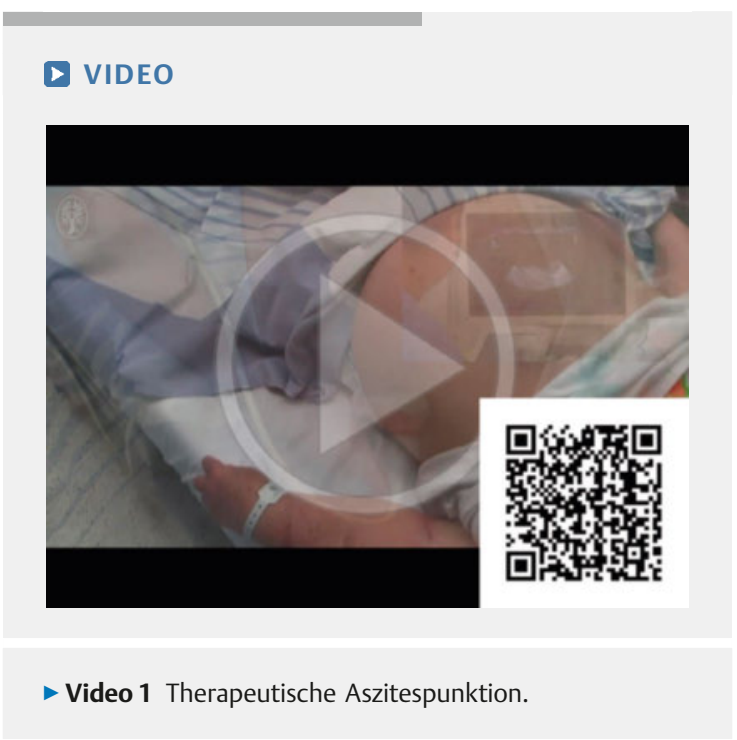




\section{Literatur}

[1] Gerbes AL, Gülberg V, Sauerbruch T et al. S3-Leitlinie Aszites, spontan bakterielle Peritonitis, hepatorenales Syndrom. Z Gastroenterol 2011; 49: 749-779

[2] Mallory A, Schaefer JW. Complications of diagnostic paracentesis in patients with liver disease. JAMA 1978; 239: 628-630

[3] Arnold C, Haag K, Blum HE et al. Acute hemoperitoneum after large-volume paracentesis. Gastroenterology 1997; 113: 978982

[4] De Gottardi A, Thevenot T, Spahr L et al. Risk of complications after abdominal paracentesis in cirrhotic patients: a prospective study. Clin Gastroenterol Hepatol 2009; 7: 906-909

[5] McVay P, Toy PT. Lack of increased bleeding after paracentesis and thoracocentesis in patients with mild coagulation abnormalities. Transfusion 1991; 31: 164-171
[6] Minocha A. A fatal case of paracentesis. Am J Gastroenterol 1999; 94: 856

[7] Eberst ME, Berkowitz LR. Hemostasis in renal disease: pathophysiology and management. Am J Med 1994; 96: 168

[8] Molino D, De Lucia D, Gaspare De Santo N. Coagulation disorders in uremia. Semin Nephrol 2006; 26: 46

[9] Gines A, Fernandez-Esparrach G, Monescillo A et al. Randomized trial comparing albumin, dextran 70 , and polygeline in cirrhotic patients with ascites treated by paracentesis. Gastroenterology 1996; 111: 1002-1010

\section{Bibliografie}

DOI https://doi.org/10.1055/s-0043-119373

Intensivmedizin up2date 2017; 13: 355-362

(c) Georg Thieme Verlag KG Stuttgart · New York

ISSN 1614-4856 